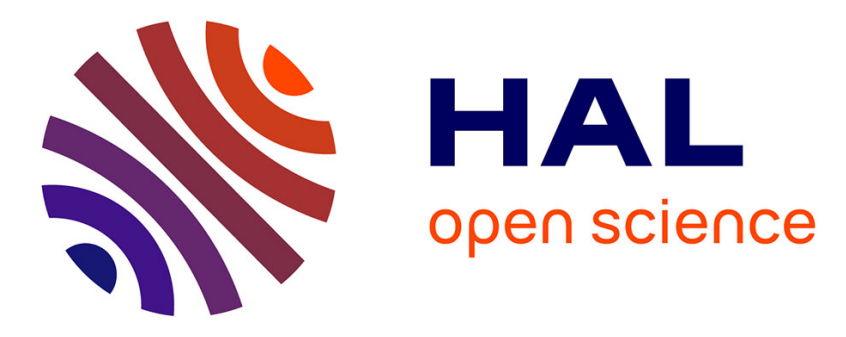

\title{
Douleurs latérales mécaniques du genou
}

Gérard Chalès, Jean-David Albert, Raphaël Guillin

\section{To cite this version:}

Gérard Chalès, Jean-David Albert, Raphaël Guillin. Douleurs latérales mécaniques du genou. Revue du Rhumatisme monographies, 2016, Pathologies du genou, 83 (3), pp.138 - 143. 10.1016/j.monrhu.2016.03.006 . hal-01376259

\section{HAL Id: hal-01376259 \\ https://hal-univ-rennes1.archives-ouvertes.fr/hal-01376259}

Submitted on 7 Oct 2016

HAL is a multi-disciplinary open access archive for the deposit and dissemination of scientific research documents, whether they are published or not. The documents may come from teaching and research institutions in France or abroad, or from public or private research centers.
L'archive ouverte pluridisciplinaire HAL, est destinée au dépôt et à la diffusion de documents scientifiques de niveau recherche, publiés ou non, émanant des établissements d'enseignement et de recherche français ou étrangers, des laboratoires publics ou privés. 
Douleurs latérales mécaniques du genou

Mechanical lateral knee pain

A paraître dans Revue du rhumatisme monographies (2016), http://dx.doi.org/10.1016/j.monrhu.2016.03.006

Gérard Chalès $^{\text {ab }}$, Jean-David Albertac, Raphaël Guillin ${ }^{\mathrm{d}}$

a) Service de rhumatologie, Hôpital sud, CHU de Rennes, 16, boulevard de Bulgarie, BP 90347, 35203 Rennes cedex 2, France

b) Université de Rennes 1,2 Avenue du Professeur Léon Bernard, 35043 Rennes, France

c) Inserm UMR 991, Hôpital Pontchaillou, 2, rue Henri Le Guilloux 35000 Rennes, France

d) Service de radiologie et imagerie ostéoarticulaire, Hôpital sud, $\mathrm{CHU}$ de Rennes, 16, boulevard de Bulgarie, BP 90347, 35203 Rennes cedex 2, France

Auteur principal et correspondant

Gérard Chalès, service de rhumatologie, Hôpital sud, CHU de Rennes

E-mail : gerard.chales@chu-rennes.fr

Mots clés: douleurs latérales du genou, arthrose fémorotibiale latérale, syndrome fémoropatellaire, articulation tibiofibulaire supérieure, ménisque latéral, discoïde, syndrome de la bandelette iliotibiale, tendinopathie bicipitale, poplitée

Key words: lateral knee pain, lateral knee osteoarthritis, patellofemoral syndrome proximal tibiofibular joint, lateral meniscus tear, discoid lateral meniscus, iliotibial band syndrome, biceps femoris, popliteus syndrome. 


\section{Résumé}

Les douleurs latérales du genou constituent un motif de consultation fréquent avec de nombreuses causes. L'interrogatoire et l'examen clinique peuvent limiter les indications de l'imagerie et réduire le nombre de causes possibles expliquées par une origine articulaire (arthrose fémorotibiale latérale, syndrome fémoropatellaire, articulation tibiofibulaire supérieure, ménisque latéral, discoïde), abarticulaire (syndrome de la bandelette iliotibiale, tendinopathie bicipitale, poplitée) ou osseuse (ostéonécrose, syndrome régional douloureux complexe, fractures de fatigue). Les douleurs référées résultant d'une coxopathie, d'une radiculopathie L4 ou L5 peuvent aussi être responsables de douleurs latérales du genou.

\section{Summary}

Lateral Knee pain is a common presenting complaint with many possible causes. History and physical examination can decrease imaging indication and narrow the possible causes explained by an articular (lateral knee osteoarthritis, patellofemoral syndrome, proximal tibiofibular joint, lateral meniscus tear, discoid lateral meniscus), a musculotendinous (iliotibial band syndrome, biceps femoris, popliteus syndrome) or an osseous origin (osteonecrosis, complex regional pain syndrome, stress fracture...). Referred pain resulting from hip joint pathology, L4 or L5 radiculopathy also may cause lateral knee pain. 


\section{Introduction}

Les douleurs mécaniques de la face latérale du genou constituent un problème de pratique quotidienne pour le rhumatologue, qu'il s'agisse d'affections articulaires (fémorotibiale, fémoropatellaire, tibiofibulaire, méniscale), osseuses (ostéonécroses, fissures osseuses, syndrome régional douloureux complexe parcellaire), abarticulaires, plus spécifiques aux sportifs (syndrome de la bandelette iliotibiale, tendinopathie bicipitale, poplitée) ou neurologiques (douleurs projetées, syndrome canalaire du nerf fibulaire commun) [1]. C'est dire la primauté de l'examen clinique, qui devrait éviter le recours systématique à l'imagerie. Sans oublier que le genou fait l'objet d'une surcote médiatique depuis la (re)découverte d'un nouveau ligament du genou, dit antérolatéral, pouvant expliquer la persistance de douleurs latérales du genou après reconstruction du ligament croisé antérieur [2].

\section{Démarche diagnostique}

Le fil conducteur est anatomique: s'agit-il d'une douleur d'origine articulaire (présence d'un épanchement, diminution de la mobilité), abarticulaire (douleur à la palpation, à l'étirement actif et passif, à la contraction contrariée), osseuse (sémiologie pauvre, données de l'imagerie), neurologique ou autre (douleur projetée ou référée) ?

L'interrogatoire est essentiel: âge de début, épisodes identiques antérieurs, antécédents traumatiques ou d'hypersollicitation articulaire, topographie et mode d'apparition de la douleur, notion de douleurs brèves et brutales ou de dérobements, activités professionnelles, activités sportives, notamment répétitives (course à pied, cyclisme), conditions de la pratique sportive, mode évolutif des douleurs [1].

L'examen clinique (voir Legré $V$ et al. [3] « dans ce numéro ») se fait debout, à la marche, puis couché. L'examen recherche un défaut d'axe du genou, dans le plan axial et sagittal en position debout, et une accentuation de la déviation à la marche (valgum dynamique), en intégrant l'examen simultané des hanches, des chevilles et de l'arrière-pied. L'inspection peut montrer un épanchement ou un empâtement du genou, la présence de cicatrices, une amyotrophie. L'examen clinique couché précise l'état des articulations fémorotibiale, fémoro-patellaire, tibiofibulaire, des ligaments et des ménisques, des tendons (tous palpables au genou), quantifie les 
mobilités, recherche un épanchement rotulien (signe du choc rotulien), et une tuméfaction palpable.

L'imagerie n'est pas systématique, en dehors de l'échographie permettant une confirmation de l'orientation clinique, essentiellement pour la pathologie abarticulaire, et la réalisation d'un geste infiltratif [4-5].

\section{Douleurs d'origine articulaire}

\subsection{Arthrose fémorotibiale latérale (AFTL)}

La fréquence de l'arthrose fémoro-tibiale latérale (AFTL) a été estimée entre 7 et $10 \%$ des cas de gonarthrose, plus fréquente chez les afro-américains et les chinois que chez les caucasiens, plus fréquente chez la femme avec un morphotype en valgus [6].

Cependant, la probabilité d'une atteinte du compartiment médial est dix fois plus importante que celle du compartiment latéral; en effet, dans la majorité de la population, l'alignement du membre inférieur est légèrement en varus $\left(1-2^{\circ}\right)$, et lors de la marche, les forces transmises au genou sont inégalement réparties entre les deux compartiments: de 60 à $80 \%$ de la charge totale est située sur le compartiment médial, soit 2,2 fois plus que le chargement du compartiment latéral [7].

Toute déformation qui déporte latéralement l'axe mécanique du membre inférieur augmente la charge du compartiment latéral du genou (coxa valga, valgisation distale du fémur, proximale du tibia, torsion tibiale externe, valgus de l'arrière-pied) ; le risque relatif de progression de l'AFTL (pincement de l'interligne suivi 6 ans) est de 6 (IC95\% 1,6-15,1) pour un morphotype en valgus comparé à des genoux normoaxés. Un IMC entre 25 et $30 \mathrm{~kg} / \mathrm{m}^{2}$ est associé à un risque relatif de progression radiologique de la gonarthrose de 2,4 (IC 1,0-3,6) comparé à un IMC inférieur à 25 et ce d'autant plus qu'il existe un défaut d'axe des membres inférieurs [6].

Le diagnostic précoce d'une AFTL se pose le plus souvent devant l'apparition de douleurs mécaniques en terrain plat chez des patients âgés de plus de 50 ans, notamment chez les femmes au décours de la ménopause; le diagnostic est confirmé par un examen radiographique standard (tableau 1); celui-ci peut être normal chez des sujets plus jeunes (avant 50 ans), notamment sportifs de bon niveau [8]. II faut rechercher la notion de pratique intensive d'activités sportives, d'antécédents traumatiques, soit une fracture du plateau tibial latéral (incidence d'AFTL radiologique rapportée de 17 à $32 \%$ ), soit une méniscectomie latérale plus arthrogène qu'une méniscectomie médiale [6].

\subsection{Syndrome fémoropatellaire}


II est fréquent, touchant un quart de la population générale (adolescents et adultes jeunes actifs), cause la plus fréquente de gonalgie (25-40\%) dans la consultation de médecine du sport, surtout chez les coureurs à pied (16-25\%). II se manifeste par des douleurs antérieures, diffuses, le plus souvent médiales mais aussi rétropatellaires et latérales, reproduites lors des activités augmentant les contraintes sur l'articulation fémoropatellaire ou répétitives ; une raideur de la bandelette iliotibiale (voir ci-dessous) dont les fibres distales se combinent à celles du rétinaculum latéral peut contribuer à une bascule latérale de la patella et une hyperpression sur l'articulation fémoropatellaire latéral [9], voir Tamalet et al. [10] « dans ce numéro ». L'arthro-scanner ou l'arthro-IRM n'est pas systématique mais reste utile pour évaluer le cartilage, l'os et la dysplasie fémoropatellaire (Fig. 1).

3.3 L'articulation tibiofibulaire supérieure: le $4^{\text {ème }}$ compartiment du genou souvent méconnu

Les douleurs de la face externe et postérieure du genou peuvent être en rapport, notamment chez le sportif, avec une laxité chronique : si la forme post-traumatique (adulte) est la plus connue, la forme spontanée (adolescent) commence à être reconnue, compte tenu d'une hypersollicitation mécanique de l'articulation tibiofibulaire qui dissipe les forces de torsion appliquées à la cheville, absorbe les moments de flexion du tibia latéral, et reçoit $1 / 6^{\text {ème }}$ du poids du corps [11] ; elle est plus fréquente en cas d'hyperlaxité associée.

L'instabilité tibiofibulaire reste rare, due à l'interligne horizontal et/ou à une implantation tibiale postérieure de la surface articulaire (2/3 des cas), et à un ligament tibiofibulaire antérieure mince; l'articulation tibiofibulaire supérieure communique avec celle du genou dans 10 à $63 \%$ des cas. La symptomatologie est peu spécifique, associant douleurs, sensation d'instabilité, signes déficitaires. La douleur touche la face latérale du genou irradiant parfois à la loge antéro-externe de jambe ou à la cuisse ( \pm raideur des ischio-jambiers), apparaissant à la marche ou à la course, majorée lors de l'impulsion ou de la réception d'un saut. La sensation d'instabilité ou de dérobement apparaît lors de la flexion maximale du genou. Des signes déficitaires sensitivomoteurs dans le territoire du nerf fibulaire commun (voir Pécourneau et al. [12] «dans ce numéro») peuvent être révélateurs, orientant vers une étiologie traumatique ou un kyste compressif (Fig. $2 \mathrm{~A}, \mathrm{~B})$ à rechercher par la palpation [13].

L'examen, sur genou fléchi à $90^{\circ}$, muscles relâchés, recherche un tiroir péronier (mobilisation antéropostérieure de la tête du péroné), indolore ou douloureux en hyperflexion du genou associée à une rotation latérale (tiroir antérieur) ou médiale (tiroir postérieur) du squelette jambier, pouvant traduire une arthropathie débutante. II y a une corrélation entre la présence d'une arthropathie dégénérative de la tibiofibulaire et celle d'une arthropathie fémorotibiale sévère, pouvant expliquer des douleurs latérales persistante après prothèse totale du genou. 
Les radiographies standard comparatives ( 2 genoux de face, de profil en extension puis en flexion dorsale et plantaire de la cheville) ne permettent pas toujours de mettre en évidence un déplacement de la tête de la fibula, nécessitant parfois un scanner pour affiner les renseignements obtenus sur le cliché de profil. L'IRM n'a d'intérêt que s'il existe une tuméfaction palpable ou repérée par l'échographie, permettant le diagnostic de kyste synovial, de kyste mucoïde (ganglion cyst), intraarticulaire (rare), de kyste intraneural ou adventitiel (artére tibiale antérieure), intramusculaire (rare), méniscal.

En cas de forme spontanée ou idiopathique, le traitement repose sur des infiltrations intraarticulaires de corticoïdes sous échographie [11] et par la mise ne place d'un strapping non compressif [13].

\subsection{Pathologie du ménisque latéral}

Pourquoi le ménisque latéral est-il plus fragile que le ménisque médial alors qu'à la marche la charge se concentre d'avantage sur le compartiment médial que sur le compartiment latéral ? L'explication vient de l'anatomie et de la biomécanique ; le ménisque latéral, plus mobile, contribue beaucoup à la congruence du compartiment latéral, notamment lors des mouvements de translation antéropostérieure survenant au cours de la flexion du genou, et est parfois le siège d'anomalies congénitales (ménisque discoïde et dérivés) pouvant favoriser la dégénérescence cartilagineuse [14]. En pratique, une méniscectomie latérale est plus arthrogène qu'une méniscectomie médiale: les analyses en éléments finis ont montré que $51 \%$ de la charge axiale totale passe par le ménisque latéral vs $30 \%$ pour le ménisque médial [6].

L'atteinte méniscale se traduit par une gonalgie centrée sur l'interligne fémorotibial survenant soit à la suite d'un traumatisme $(20 \%)$, soit de manière progressive (origine dégénérative, $80 \%$ ) [15] ; en cas de méniscose dégénérative, il n'y a pas de blocages et les douleurs sont aggravées par la montée des escaliers et la position accroupie.

L'examen clinique révèle une douleur à la pression de l'interligne latéral dans une zone très limitée (plus diffuse dans la gonarthrose), un épanchement d'importance variable, une mobilité généralement normale parfois douloureuse en flexion ou en extension forcées, un signe de Mac Murray positif ; il est indispensable de faire un examen complet du genou et de vérifier l'état du pivot central [voir Legré et al. [3] " dans ce numéro »]

La réalisation de clichés radiographiques standard du genou est systématique, le plus souvent normaux ; la réalisation d'une IRM [Fig. 3] peut aider dans la démarche diagnostique (tableau un peu atypique ou laissant planer un doute sur une éventuelle ostéonécrose aseptique chez un patient de plus de 60 ans) et thérapeutique. 
Le traitement est médical en première intention (antalgiques et AINS) et s'il existe un point douloureux précis sur l'interligne latéral, on peut proposer une infiltration juxtaméniscale, même s'il existe une arthrose débutante très fréquemment associée, véritable test diagnostique quant à la responsabilité ou non des lésions méniscales ; elle ne doit pas être réalisée (sans point gâchette) uniquement sur la vue des lésions méniscales à l'IRM, présentes chez $25 \%$ de sujets de plus de 50 ans asymptomatiques [15-16]. Cette infiltration, efficace dans $75 \%$ des cas, permet d'éviter l'arthroscopie avec réparation méniscale ou méniscectomie partielle [14-15].

Le ménisque discoïde représente une anomalie rare $(0,4$ à $17 \%$ de la population) du ménisque latéral, parfois bilatéral ( $8 \%$ ), affection congénitale la plus fréquente chez l'enfant, dont la classification repose sur la proportion de surface couverte du plateau tibial (complet, incomplet, type ligament de Wrisberg incriminé dans le ménisque hypermobile) ; associé à des lésions méniscales dans $3 / 4$ des cas (fissures horizontales centrales et lésions verticales périphériques postérieures) et à une ostéochondrite du condyle latéral chez le sujet jeune [14].

Le claquement sec, classique « snapping knee syndrom », non spécifique (tableau 2) [17] est présent une fois sur deux, tout comme le syndrome méniscal classique (blocages, épanchement, instabilité). La palpation peut révéler un claquement sourd « clunk syndrom » (1/4) lors du retour vers l'extension vers 15-20. Les tests méniscaux sont positifs une fois sur deux [14].

Les radiographies standard sont peu contributives au diagnostic. L'IRM est l'examen de référence permettant le diagnostic [Fig. 4] et la recherche de lésions associées [18]. Le traitement ne concerne que les ménisques discoïdes symptomatiques ou compliqués: méniscoplastie partielle sous arthroscopie permettant une économie méniscale, avec de bons résultats à long terme [19].

Le kyste du ménisque latéral du genou est l'association d'une lésion méniscale (clivage horizontal) et d'une tuméfaction de l'interligne latéral dont l'incidence varie de 1,2 à $1,5 \%$, souvent d'origine dégénérative, parfois post-traumatique $(30 \%)$, survenant chez des sujets jeunes, sportifs (2/3) [14]. Ces kystes résultent le plus souvent d'une fissuration méniscale sous-jacente en notant que, si la valeur prédictive positive du kyste avoisine $90 \%$ à $100 \%$ selon les séries au sein du genou, cette valeur n'est que de $64 \%$ au sein de la corne antérieure du ménisque latéral [20].

II se manifeste par une douleur du segment moyen de l'interligne, avec des signes méniscaux et la présence d'une tuméfaction palpable. L'IRM permet le diagnostic positif et différentiel, et guide la stratégie thérapeutique. L'arthroscopie est indispensable (méniscectomie ou suture méniscale) [14].

\section{Douleurs d'origine abarticulaire}




\subsection{Syndrome de la bandelette iliotibiale}

La bandelette iliotibiale est un épaississement du fascia lata, s'étendant du grand trochanter au tubercule de Gerdy, avec des ramifications vers le ligament fémoropatellaire latéral. Cette bande fibreuse pourrait soit frotter sur le condyle fémorale latéral [Fig 1, matériel supplémentaire] à chaque flexion-extension du genou (mécanisme de cisaillement par mobilité antéropostérieure), d'où le nom de syndrome de l'essuie-glace, soit comprimer les structures sous-jacentes (tissu graisseux) lors de la flexion du genou à $30^{\circ}$ (mécanisme de compression par mobilité latéromédiale) ou encore donner lieu à une enthésopathie d'insertion sur le condyle fémoral (mécanisme de traction) [21].

Le syndrome de la bandelette iliotibiale (SBIT) est la tendinopathie la plus fréquente du compartiment latéral, déclenché par la course à pied (incidence de 5 à 14\%) [22], la marche en terrain varié (randonnée), le cyclisme (incidence de 15 à 24\%) ; plusieurs facteurs ont été incriminés: un déficit musculaire des abducteurs de hanche, les facteurs biomécaniques (cinétique de la course), le type de chaussures et le type de revêtement, sans aucune conclusion définitive concernant la responsabilité de ces facteurs [22]. Serait plus exposé le sujet masculin, de niveau moyen, ayant repris la course depuis quelques mois avec un à deux entraînements hebdomadaires (changement des habitudes de la pratique sportive) [1].

Le SBIT se traduit par une douleur initialement diffuse de la face latérale du genou puis plus localisée au condyle au décours puis au cours de la course à pied (entraînant l'arrêt de la course), parfois au repos, aggravée par les descentes, l'allongement de la foulée ou la station assise prolongée avec le genou fléchi. L'arrêt des activités sportives n'est pas toujours efficace sur la symptomatologie douloureuse [1].

L'insertion distale de la bandelette iliotibiale sur le condyle latéral est douloureuse 2 à $3 \mathrm{~cm}$ au-dessus de l'interligne latéral lors d'une flexion passive passant de $90^{\circ} \mathrm{à} 30^{\circ}$ (test de Noble). La douleur peut être reproduite lors de mouvements de flexionextension du genou de $30^{\circ}$ d'amplitude, en appui monopodal (test de Renne). Une rétraction du fascia lata peut être recherchée par le test d'Ober sur un patient en décubitus latéral sur le côté sain, hanche et genou sains à $90^{\circ}$ de flexion: le patient a des difficultés à réaliser une adduction de la cuisse (genou à $90^{\circ}$ ) au-delà de la ligne médiane, avec apparition d'une douleur de la face latérale du genou [21].

L'imagerie n'a pas d'intérêt dans les formes typiques, en dehors de l'échographie dynamique ; en effet celle-ci a permis de démontrer - chez des coureurs à pied asymptomatiques - que la bandelette iliotibiale a une mobilité antéropostérieure par rapport au condyle latéral dans l'arc de mobilité $0-45^{\circ}$ en charge et en décharge combinée à une réduction de sa largeur [23], associée à un épanchement liquidien sous la bandelette dont la présence varie selon la position (95\% en charge et en extension vs $22 \%$ à $30^{\circ}$ de flexion), en rapport possible avec une extension du récessus latéral synovial [24]: cet épanchement n'est donc pas toujours synonyme 
d'un SBIT. En pratique, la sensibilité de l'échographie dans le bilan d'un SBIT est faible. L'échographie peut constituer un outil diagnostique non spécifique permettant une évaluation dynamique des tendons, ainsi qu'une aide à la planification et au suivi thérapeutique (indication et réalisation d'une infiltration) [21] ; elle ne permet pas le diagnostic des lésions intrarticulaires associées.

Le traitement est médico-rééducatif $[21,25]$, comportant à la phase aiguë repos strict, application locale de froid et d'AINS; en cas d'échec, on peut proposer une infiltration locale de corticoïdes réalisée soit loco dolenti, soit sous échographie [Fig. 2-3, matériel supplémentaire] permettant de mieux cibler l'espace entre la bandelette et le condyle (zone hypo-échogène) et/ou de repérer le récessus latéral ; ce geste a une efficacité transitoire [1,26]. C'est dire qu'il faut envisager secondairement des étirements (tenseur du fascia lata, muscles sous-pelviens) [27] et un renforcement musculaire (moyen fessier, quadriceps) [21,25] ; les massages transverses profonds ne sont pas recommandés selon une revue Cochrane récente [28]. Enfin, une réadaptation progressive de la course à pied est indispensable, en modifiant les paramètres (reprise sur un temps bref à un rythme soutenu, terrains plats, chaussures avec semelles correctrices) $[1,21,25]$. II existe de rares formes réfractaires nécessitant un bilan IRM [Fig. 3-4, matériel supplémentaire] et/ou une arthroscopie (lésions intraarticulaires intriquées) puis un abord chirurgical direct (incision ou résection de la bandelette, plastie en Z) [29].

\subsection{Tendinopathie bicipitale}

Le tendon du biceps fémoral s'insère essentiellement sur le sommet de la tête fibulaire puis sur la face latérale de l'épiphyse proximale du tibia, avec une expansion aponévrotique pour le fascia lata. Les faisceaux tendineux sont séparés du ligament collatéral latéral par une bourse. Les tendinopathies du biceps fémoral sont rares. Trois formes cliniques ont été décrites :

- La ténosynovite touche les coureurs cyclistes, attribuée à un mauvais programme d'entraînement et à l'utilisation d'un matériel mal adapté. La douleur est surtout exacerbée par l'étirement et la palpation de la tête fibulaire (parfois crépitation) [1]. Le traitement est celui de toute tendinopathie (repos, AINS) mais nécessite la vérification technique du vélo, très rarement une infiltration péritendineuse sous échographie [25].

- La forme fissuraire, lésion chronique de surmenage, touche les coureurs à pied, les haltérophiles ou adeptes de la musculation ou des sports collectifs, avec un début parfois brutal, le trépied de la douleur, une impotence fonctionnelle (impossibilité de courir) nécessitant un bilan échographique et l'arrêt des activités sportives nocives [1] ;

- Le syndrome du ressaut du biceps fémoral est visible au niveau de la tête du péroné entre $110^{\circ}$ et $120^{\circ}$ de flexion, entraînant des douleurs après flexions répétées du genou et après effort (tête du péroné) [30]. La radiographie 
recherche une hypertrophie de la tête du péroné ou une anomalie de la tibiofibulaire supérieure ; l'échographie permet l'étude de l'extrémité distale du biceps, la recherche d'une anomalie d'insertion à type de faisceau tibial variant, expliquant souvent le ressaut, et la responsabilité du faisceau (notamment tibial) à l'origine du ressaut [31].

\subsection{Tendinopathie poplitée}

Le tendon poplité a un trajet final intra-articulaire. La tendinopathie est rare et il n'est pas toujours facile de la distinguer du SBIT. La douleur est diffuse postérolatérale, survenant lors d'appuis pendant la course, notamment en descente, aggravée par les changements de direction [25] ; elle est située au-dessus de l'interligne latéral, en avant du ligament latéral, réveillée sur un patient en décubitus dorsal par la mise de la hanche en flexion-abduction-rotation externe, genou fléchi à $90^{\circ}$. Il existe souvent une hypermobilité postérolatérale. Le traitement nécessite une remusculation des rotateurs du genou et un travail proprioceptif [25].

\section{Douleurs d'origine osseuse}

Devant une gonalgie d'apparition brutale, à radiographie normale, il faut évoquer

le diagnostic d'ostéonécrose spontanée (post fracture sous-chondrale ; voir Lafforgue $P$ [32] "dans ce numéro"), secondaire (femme, < 45 ans, atteinte condylienne bilatérale) ou post-méniscectomie (prévalence très faible, homme et femme $>45$ ans, territoire adjacent au geste méniscal). L'ostéonécrose spontanée du condyle latéral est très rare $(<5 \%)$, touchant la femme mais aussi l'homme d'âge mûr, aggravée par le valgus [33] ;

le diagnostic d'algodystrophie parcellaire, avec des signes pseudoinflammatoires apparents ou discrets, et une douleur élective et intense à la palpation du condyle ou du plateau tibial [34], confirmée par l'IRM ;

Une fracture de fatigue du col du péroné ou de l'extrémité supérieure du tibia [27] ;

L'imagerie (IRM) peut révéler des lésions osseuses non visibles sur les radiographies standards ou déjà visibles (ostéonécrose, ostéochondrite disséquante, tumeurs osseuses ...).

\section{Douleurs projetées}

S'il n'existe pas de causes régionales pour expliquer les douleurs latérales, il faut penser à la possibilité d'une coxopathie qui peut se révéler par une douleur du genou, à une radiculalgie L4 ou L5 par hernie discale ou sténose lombaire. 


\section{Conclusions}

La face latérale du genou est le siège de nombreuses douleurs mécaniques d'origine articulaire, osseuse non spécifiques et abarticulaire (plus spécifique des sportifs) nécessitant un bilan clinique soigneux et un bilan d'imagerie si le diagnostic lésionnel est incertain.

Déclaration d'intérêts : Les auteurs ne déclarent aucun conflit d'intérêt.

\section{Références}

1. Rodineau J. Les douleurs latérales de la face latérale du genou chez les sportifs. J Traumatol Sport 2010;27:1-3.

2. Lubowitz JH, Provencher MT, Brand JC, Rossi MJ. The knee anterolateral ligament. Arthroscopy 2014;30:1385-8.

3. Legré V, Boyer T. L'examen clinique du genou. Rev Rhum Monogr 2015.

4. Albert JD, Marin F. Intérêt diagnostique et thérapeutique de l'échographie du genou. Rev Rhum 2006;73:625-32.

5. De Maeseneer M, Marcelis S, Boulet C, Kichouh M, Shahabpour M, de Mey J et al. Ultrasound of the knee with emphasis on the detailed anatomy of anterior, medial, and lateral structures. Skeletal Radiol 2014;43:1025-39.

6. Scott CE, Nutton RW, Biant LC. Lateral compartment osteoarthritis of the knee:Biomechanics and surgical management of end-stage disease. Bone Joint J 2013;95-B:436-44.

7. Mündermann A, Dyrby CO, D'Lima DD, Colwell CW, Andriacchi TP. In vivo knee loading characteristics during activities of daily living as measured by an instrumented total knee replacement. J Orthop Res 2008;26:1167-72.

8. Chevalier X. Diagnostic précoce de la gonarthrose. Rev Prat 2009;59:1243-9.

9. Petersen W, Ellermann A, Gösele-Koppenburg A, Best R, Rembitzki IV, Brüggemann GP et al. Patellofemoral pain syndrome. Knee Surg Sports Traumatol Arthrosc 2014;22:2264-74.

10. Tamalet $B$, Rochcongar $P$, Rochcongar $G$. La fémoropatellaire, une articulation oubliée ? Rev Rhum Monogr 2015.

11. Smith J, Finnoff JT, Levy BA, Lai JK. Sonographically guided proximal tibiofibular joint injection: technique and accuracy. J Ultrasound Med 2010;29:783-9.

12. Pecourneau V, Rochcongar $P$, Cintas $P$. Syndromes canalaires du genou. Rev Rhum Monogr 2015.

13. Manet MP, Lasbleiz S, Bizot P. Articulations péronéo-tibiales. In: Kahn MF, Kuntz D, Meyer O, Bardin T, Orcel P. L'actualité rhumatologique 2001. Paris: Elsevier; 2001. p. 176-91.

14. Beaufils $P$, Hardy $P$. Le ménisque latéral de l'adulte. Rev Chir Orthop 2006;92(suppl):2S169-94. 
15. Bouvard $M$, Juret $A$. Intérêt des infiltrations du « mur méniscal » par les corticoïdes dans les douleurs méniscales du genou. A propos de 214 cas. J Traumatol Sport 2008;25:4-10.

16. Lequesne $M$. Le foyer de périméniscite mérite bien son infiltration : du concept à la pratique de la juxtaméniscale. J Traumatol Sport 2008;25:123-4.

17. Marchand AJ, Proisy M, Ropars M, Cohen M, Duvauferrier R, Guillin R. Snapping knee: imaging findings with an emphasis on dynamic sonography. AJR 2012;199:142-50.

18. Jellouli O, Slioui B, Amil T, Ennouali H. Ménisque discoïde. Feuil Radiol 2015;55:120-22.

19. Chedal-Bornu B, Morin V, Saragaglia D. Meniscoplasty for lateral discoid meniscus tears: Long-term results of 14 cases. Orthop Traumatol Surg Res 2015;10:699-702.

20. De Smet AA, Graf BK, del Rio AM. Association of parameniscal cysts with underlying meniscal tears as identified on MRI and arthroscopy. AJR 2001;196: W180-6.

21. Hirt D. Le syndrome de l'essuie-glace. Mise au point. Réflexions Rhumatologiques 2015;19:5-9.

22. van der Worp MP, van der Horst N, de Wijer A, Backx FJ, Nijhuis-van der Sanden MW. Iliotibial band syndrome in runners: a systematic review. Sports Med 2012;42:969-92.

23. Jelsing EJ, Finnoff JT, Cheville AL, Levy BA, Smith J. Sonographic evaluation of the iliotibial band at the lateral femoral epicondyle: does the iliotibial band move? J Ultrasound Med 2013;32:1199-206.

24. Jelsing EJ, Maida E, Finnoff JT, Smith J. The source of fluid deep to the iliotibial band: documentation of a potential intra-articular source. PM R 2014;6:134-8.

25. Rochcongar $P$. Tendinopathie du genou chez le sportif. Rev Prat 2009;59:1257-60.

26. Ellis R, Hing W, Reid D. Iliotibial band friction syndrome--a systematic review. Man Ther 2007;12:200-8.

27. Fullem BW. Overuse lower extremity injuries in sports. Clin Podiatr Med Surg 2015;32:239-51.

28. Loew LM, Brosseau L, Tugwell P, Wells GA, Welch V, Shea B et al. Deep transverse friction massage for treating lateral elbow or lateral knee tendinitis. Cochrane Database Syst Rev 2014;11:CD003528.

29. Mezzadri G, Servien E, Lustig S, Neyret P. Intérêt de l'arthroscopie dans le traitement du syndrome de la bandelette iliotibiale. J Traumatol Sport 2010;27:4-9.

30. Prothoy I, Cartier JL. Ressaut distal du biceps fémoral : à propos de 2 cas et revue de la littérature. J Traumatol Sport 2014;31:101-5.

31. Guillin R, Mendoza-Ruiz JJ, Moser T, Ropars M, Duvauferrier R, Cardinal E. Snapping biceps femoris tendon: a dynamic real-time sonographic evaluation. J Clin Ultrasound 2010;38:435-7. 
32. Lafforgue P. Ostéonécrose spontanée du genou: le résultat d'une fracture sous-chondrale? Rev Rhum Monogr 2015.

33. Ohdera T, Miyagi S, Tokunaga M, Yoshimoto E, Matsuda S, Ikari H.

Spontaneous osteonecrosis of the lateral femoral condyle of the knee: a report of 11 cases. Arch Orthop Trauma Surg 2008;128:825-31.

34. Doury P, Delahaye RP, Granier R, Pattin S, Metges PJ, Fabresse F. L'algodystrophie parcellaire. Rev Rhum Mal Osteoartic 1979;46:37-44.

Tableau 1 Bilan radiographique standard pour une gonarthrose

Radiographie de face debout prenant les deux genoux

Profil à $30^{\circ}$ de flexion

Défilé fémoro-patellaire à $30^{\circ}$

Schuss (cliché postéro-antérieur)

Bassin de face

Tableau 2 Causes des ressauts de la face latérale

Tendon biceps fémoral

Tendon poplité

Bandelette iliotibiale

Ménisque

Plica

Tumeurs ou corps étrangers intraarticulaires 


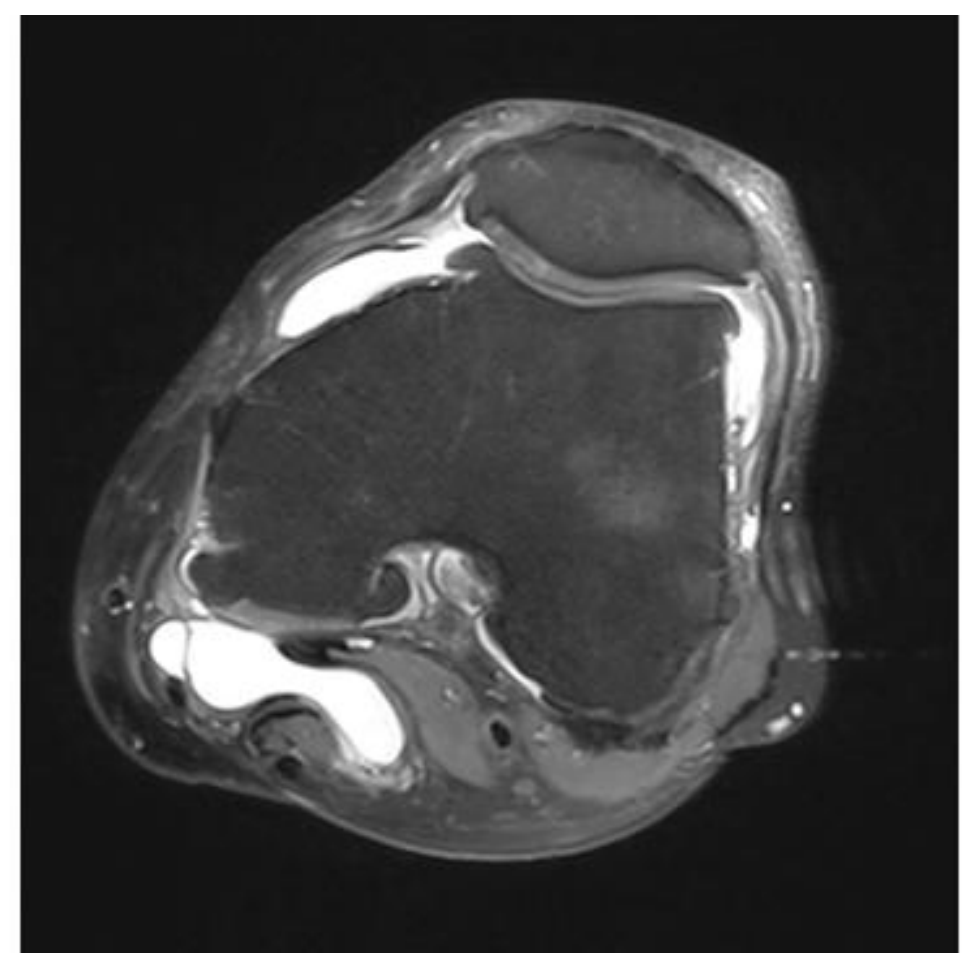

Fig. 1 IRM du genou dans le plan axial, en densité de protons avec saturation du signal de la graisse. Une arthrose fémoropatellaire est notée sous forme d'une ostéophytose marginale avec épanchement articulaire abondant. 


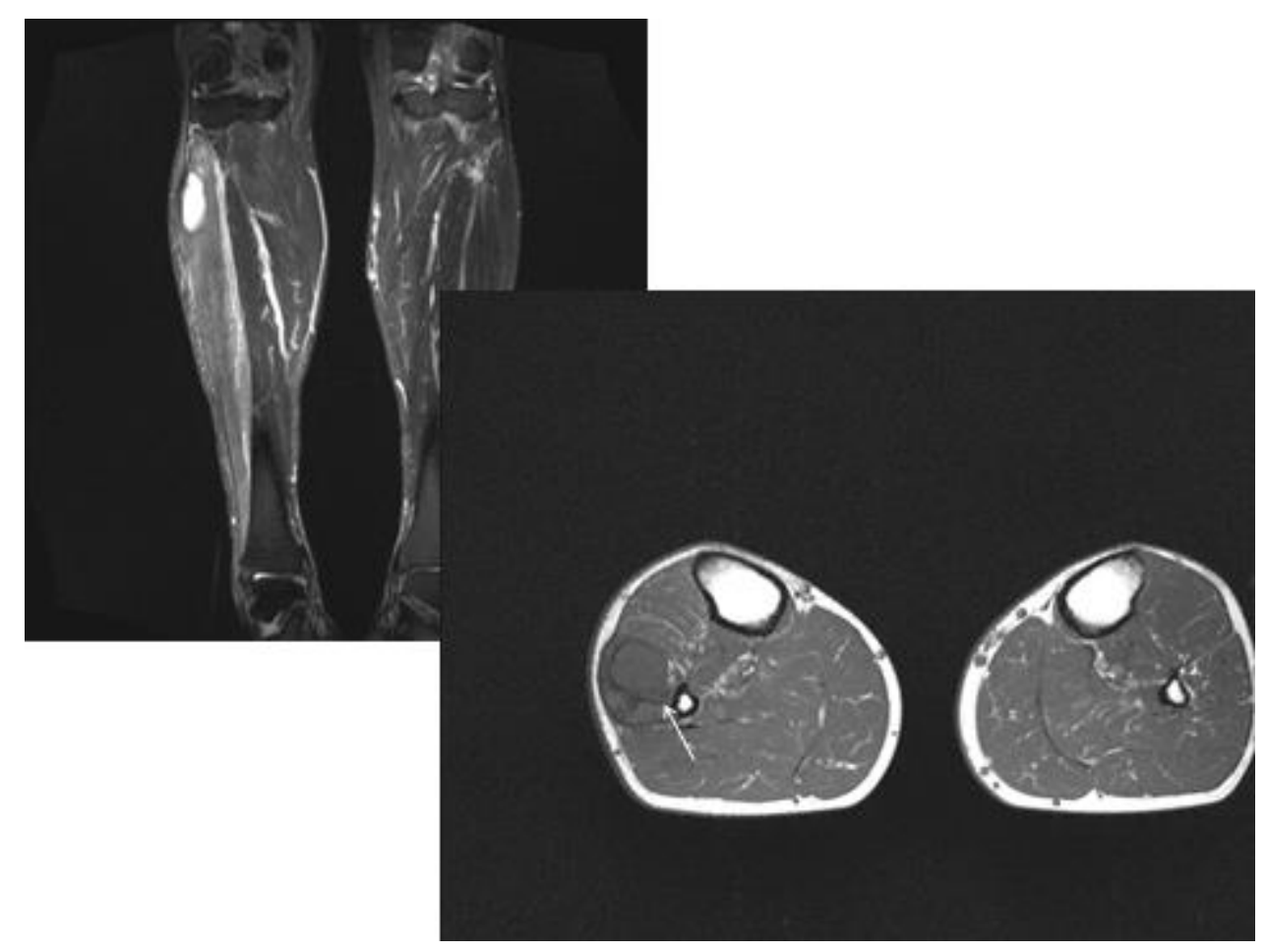

Fig. 2A IRM du genou dans le plan coronal Stir puis axial T1. 62 ans, kyste arthrosynovial tibiofibulaire compressif sur le nerf fibulaire commun (flèche). Noter la dénervation des muscles des loges antérieure et antéro-latérale dans le plan coronal, sous forme d'un hypersignal diffus de ces dernières.

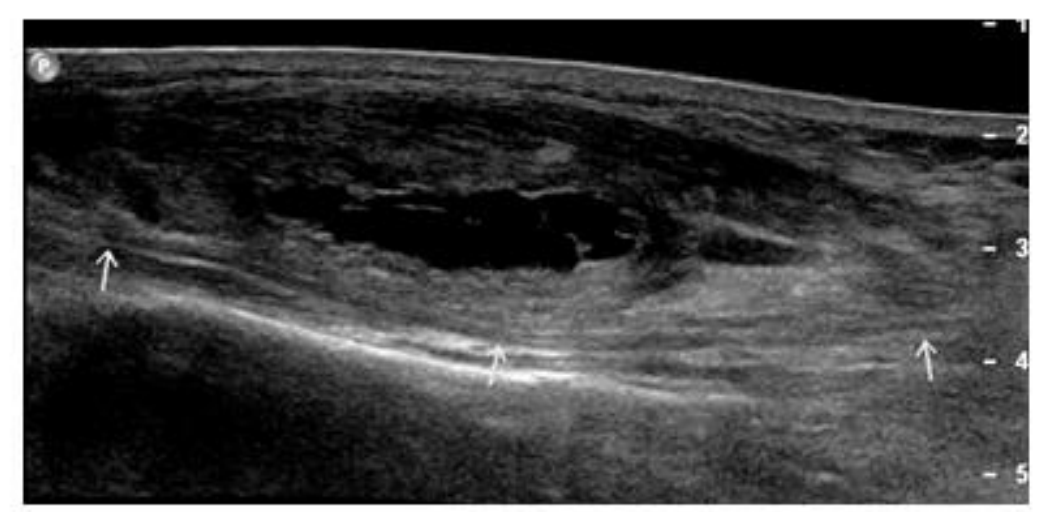

Fig 2B Echographie du genou, en coupe coronale centrée sur le kyste. Celui-ci est intra-musculaire et refoule en profondeur le nerf fibulaire commun (flèches). 


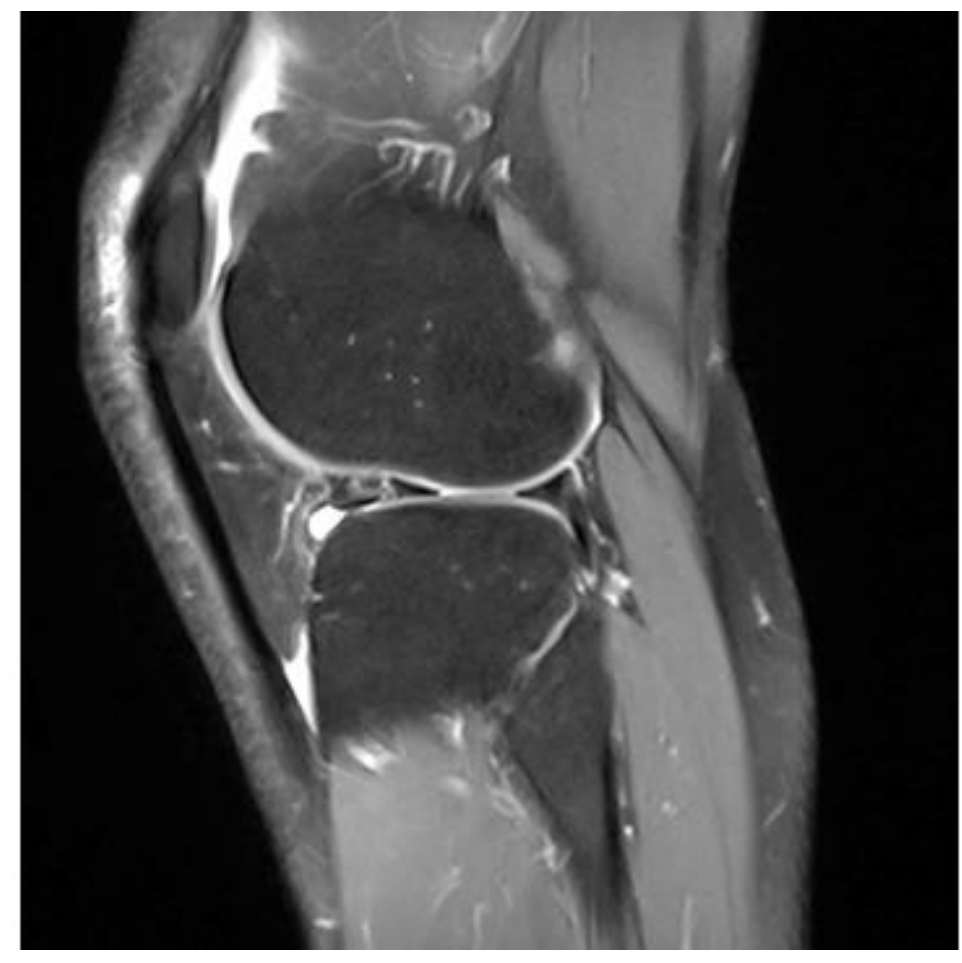

Fig. 3 IRM du genou dans le plan sagittal en densité de protons avec saturation du signal de la graisse. 44 ans, fissuration complexe de la corne antérieure du ménisque latéral (sportif). 


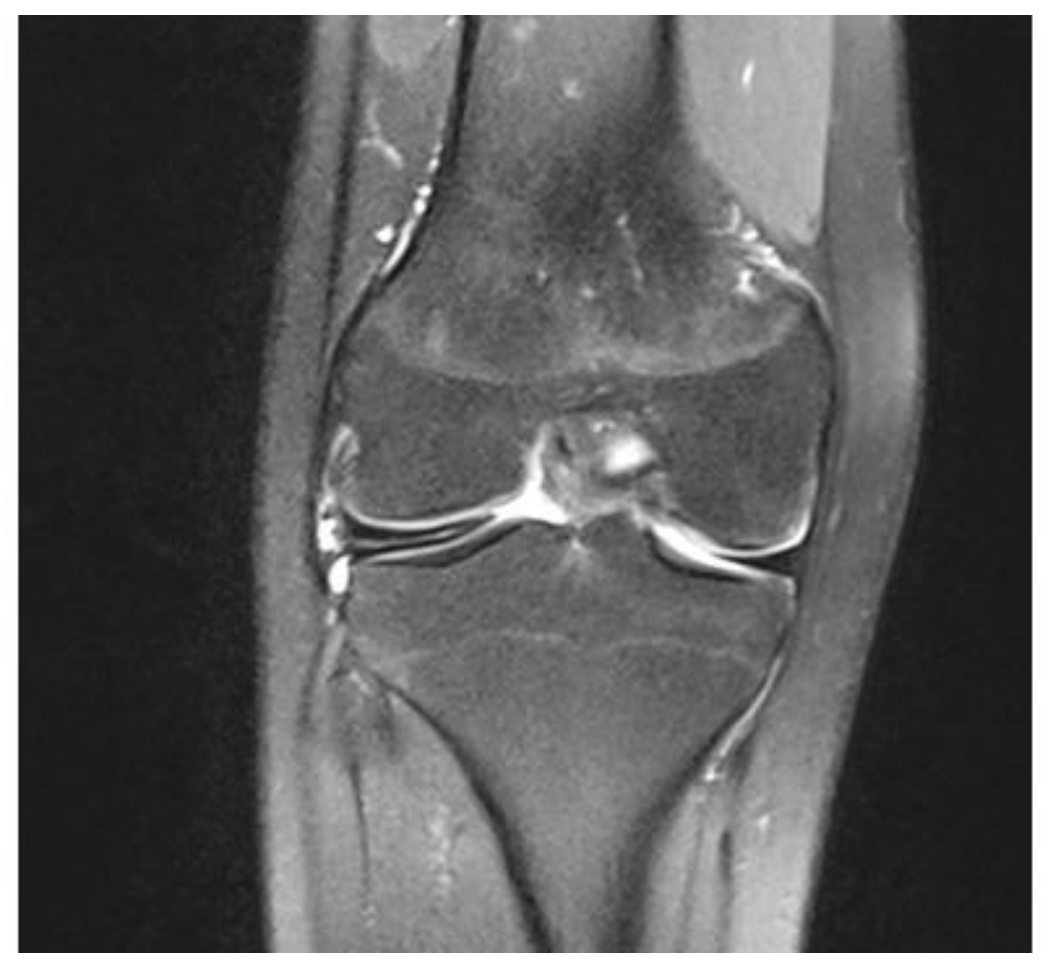

Fig. 4 IRM du genou dans le plan coronal en densité de protons avec saturation du signal de la graisse. 17 ans, fissuration d'un ménisque latéral discoïde. 